\title{
PROCESSI DI TRASFORMAZIONI URBANA E MERCATO ABITATIVO: IL CASO DELLA CITTÀ DI TORINO (ITALIA)
}

\author{
Bravi, Marina \\ marina.bravi@polito.it
}

Ricercatore e Professore presso il Dipartimento Casa-Città del Politecnico di Torino

Torino, Italia

Remisión articulo: 18-8-2006 Remisión definitiva: 1-9-2006

Parole-chiave: mercato abitativo, programmi di riqualificazione urbana (PRIU)

Sommario: Vengono delineati i rapporti tra mercato abitativo e processi di trasformazione urbana, con particolare riferimento al caso della Città di Torino (Italia). La riconversione, negli ultimi quindici anni, di più di tre milioni di mq. di aree industriali abbandonate ha costituito un'occasione unica per cambiare completamente il volto della Città e le condizioni degli abitanti. Ma la forte concentrazione degli investimenti pubblici e privati, sostenuti da una congiuntura immobiliare positiva e da un forte trend di crescita dei prezzi, ha determinato una situazione apparentemente contrastante. II forte predominio dell'offerta privata di abitazioni sul libero mercato, sia della proprietà che dell'affitto e l'erosione progressiva di quest'ultimo, in termini percentuali, hanno infatti creato una situazione di disagio abitativo che sembra contraddire alcuni obiettivi del primo Piano Strategico.

\section{Introduzione}

Torino è stata recentemente protagonista del più imponente complesso di trasformazioni urbane mai avviato a partire dal dopoguerra; mentre la Città si preparava a celebrare l'evento olimpico come uno dei più importanti della sua storia, si concludevano i grandi cantieri, il cui iter amministrativo si era avviato alla fine degli anni '90. Dal concorso di una serie di processi di trasformazione urbanistica ${ }^{1}$ e di avvenimenti di carattere eccezionale, si sono dunque create, nel volgere di pochi anni, le condizioni affinché Torino mutasse

\footnotetext{
${ }^{1}$ L'approvazione del PRG nel 1995 ha assunto un peso determinante nell'innescare la trasformazione urbana. Verso la fine degli anni ' 90 si sono poi sommati nuovi interventi, progettati nell'ambito del primo Piano Strategico e ai fini della scadenza olimpica del 2006. Sempre dagli anni ' 90 in poi, si sono concretizzate nuove modalità di intervento urbanistico, con la definizione dei cosiddetti Programmi Complessi (PC). Alcuni orientamenti erano, per altro, già contenuti nello stesso PRG, ma solo nella seconda metà degli anni '90 i PC diventano i principali strumenti di attuazione del piano. Tra il 1995 e il 2001, vengono approvati a Torino 11 Programmi di riqualificazione urbana (PRIU), 6 Programmi integrati (PRIN), 3 Programmi di recupero urbano (PRU), 8 Piani particolareggiati (PP). A questi si aggiungono gli strumenti ordinari, previsti dallo stesso PRG (16 Piani Esecutivi Convenzionati, 20 Concessioni Convenzionate) e 16 Studi Unitari d'Ambito, per consentire la realizzazione degli interventi che riguardano obbiettivi specifici del PRG. La formazione di Piani di Riqualificazione Urbana si avvia a Torino nel 1994 e il primo accordo di programma viene firmato nel luglio 1998; tutti gli altri seguono nell'arco di sei mesi, anche per l'approssimarsi della scadenza imposta dalla legge (31.12.1998). I programmi per le "Spine" 1, 3 e 4 interessano grandi aree industriali abbandonate, dando quindi sostanzialmente attuazione alle indicazioni del PRG, nonostante sia stata successivamente ridotta la loro consistenza dimensionale. Anche nei Comuni dell'area metropolitana si è assistito in questi anni a una notevole attività di pianificazione. Molte realtà municipali hanno sviluppato PC per la trasformazione del territorio, accedendo a finanziamenti nazionali e regionali. I Comuni dell'area Ovest sono, per altro, anche quelli interessati dalla nuova linea di metropolitana. Cfr. Spaziante, Ciocchetti, 2006; Mazza, 2004; Bianchetti, 2006.
} 
radicalmente il suo volto. Si è trattato di uno spettacolare laboratorio di politica urbanistica, i cui esiti, per nulla scontati, necessitano per lo meno di un resoconto, se non di un vero e proprio bilancio costi-benefici. E' necessario, in altre parole, domandarsi se ci si è avviati nella direzione auspicata - dagli amministratori come dai cittadini - o se, al contrario, la portata di determinati fenomeni, travalicanti l'ambito strettamente locale, abbia prodotto nuove disparità e contraddizioni a livello urbano.

In questo breve saggio, che necessariamente rimanda a ricerche più approfondite ${ }^{2}, \mathrm{ci}$ si occuperà pertanto di un tema di carattere trasversale - il mercato abitativo - che ha occupato e occupa una posizione privilegiata all'interno del "Piano strategico per la promozione della città di Torino", che, a partire dal febbraio 2000, ha il compito di definire le linee guida dei processi di trasformazione locale. II tema della qualità abitativa si intreccia infatti con quello della rigenerazione urbana (Ave, 2004), ambito in cui l'area metropolitana di Torino è stata, negli ultimi dieci anni, luogo di sperimentazione di interventi innovativi, proprio da questo punto di vista: dal "Progetto Periferie" ai più recenti "Contratti di Quartiere". Promuovere lo sviluppo locale ha significato infatti affrontare il problema in un'ottica integrata, coordinando interventi di tipo urbanistico ed edilizio, sociale ed economico, facendo perno sulle risorse locali. Va ricordato che l'assenza di una politica abitativa adeguata determina situazioni sociali che incidono negativamente, nel medio ma anche nel lungo periodo, sulle stesse politiche di sviluppo; la qualità abitativa fa parte di quell'insieme di condizioni localizzate che conferiscono a un'area un sicuro vantaggio competitivo, poiché si tratta di un elemento fondamentale, relativo al benessere collettivo, ma anche di una risorsa di coesione sociale.

\section{II mercato abitativo a Torino e il trend nazionale}

E' utile ricordare che Torino è, dopo Roma, Milano e Napoli, la quarta città italiana per numero di abitanti e per dimensione del mercato abitativo. Costituisce da sola, con un valore dello stock pari a 490.698 unità immobiliari, il $40,1 \%$ dell'intera sua provincia. La particolarità di questa Città è rappresentata da un'elevata densità di abitazioni per kmq. di superficie comunale, a differenza dei territori della cintura. Associato all'alta densità, vi è poi un elevato numero di abitazioni per edificio: 12,3 abitazioni a fronte di 8,6 degli altri maggiori comuni.

A Torino e provincia il mercato immobiliare residenziale ha registrato una notevole crescita nel corso degli ultimi sei anni, sia dal punto di vista del numero di transazioni che per ciò che concerne i valori. Tale fenomeno appare coerente con quanto è avvenuto a livello nazionale, ove la fase positiva - iniziata nel 1997 con un $+8,7 \%$ di immobili scambiati rispetto all'anno precedente - ha raggiunto l'apice di 883.350 transazioni normalizzate annue (NTN) ${ }^{3}$

\footnotetext{
${ }^{2}$ Questo saggio prende spunto da alcuni temi contenuti nel Programma di Rilevante Interesse Nazionale (PRIN), finanziato dal Ministero dell'Università e della Ricerca Scientifica. Interventi di trasformazione e riqualificazione urbana a Torino: la valutazione degli impatti sul mercato immobiliare. Coordinatore scientifico del Programma di Ricerca Nazionale: E. Mollica Responsabile scientifico dell'Unita' Locale di Ricerca (Torino): F. Zorzi, biennio 2004-2005. Ciò nonostante la responsabilità delle considerazioni svolte in questa sede resta unicamente dell'Autrice.

${ }^{3}$ Agenzia del Territorio, Osservatorio del Mercato Immobiliare (OMI). Con NTN si intende il numero di transazioni per quota di proprietà oggetto della transazione. Ciò significa che, ad esempio, nel caso di tre transazioni aventi per oggetto rispettivamente 1/3, 1/3 e 1 del diritto di proprietà, il NTN è 1,66 e non 3 . Mentre con IMI si intende il rapporto tra NTN e stock di unità immobiliari; esso rappresenta un indicatore di intensità del mercato.
} 
nel 2005. In particolare, l'incremento del volume di compravendite immobiliari a destinazione residenziale, nel 2005 rispetto al 1996, è risultato del 72,5\% ${ }^{4}$.

Tabella 1 - Numero di transazioni e intensità di mercato tra il 2000 e il 2005.

\begin{tabular}{|c|c|c|c|c|c|c|c|c|c|c|c|c|}
\hline \multirow{2}{*}{ Prov. } & \multicolumn{2}{|c|}{2000} & \multicolumn{2}{|c|}{2001} & \multicolumn{2}{|c|}{2002} & \multicolumn{2}{|c|}{2003} & \multicolumn{2}{|c|}{2004} & \multicolumn{2}{|c|}{2005} \\
\hline & NTN & IMI & NTN & IMI & NTN & IMI & NTN & IMI & NTN & IMI & NTN & IMI \\
\hline Torino & $17.103,54$ & $3,59 \%$ & $15.938,98$ & $3,34 \%$ & $17.093,29$ & $3,57 \%$ & $16.344,64$ & $3,37 \%$ & $16.299,44$ & $3,34 \%$ & $16.284,15$ & $3,32 \%$ \\
\hline Provincia TO & $35.895,62$ & $3,13 \%$ & $33.038,33$ & $2,83 \%$ & $36.312,81$ & $3,08 \%$ & $35.438,46$ & $2,96 \%$ & $37.236,85$ & $3,08 \%$ & $37.752,62$ & $3,09 \%$ \\
\hline Piemonte & $63.600,61$ & $2,73 \%$ & $59.146,82$ & $2,50 \%$ & $65.769,83$ & $2,74 \%$ & $65.322,47$ & $2,67 \%$ & $68.986,14$ & $2,79 \%$ & $70.468,11$ & $2,82 \%$ \\
\hline Italia & $690.478,08$ & $2,52 \%$ & $681.264,25$ & $2,44 \%$ & $761.522,17$ & $2,69 \%$ & $762.085,87$ & $2,64 \%$ & $804.125,66$ & $2,75 \%$ & $833.349,80$ & $2,80 \%$ \\
\hline
\end{tabular}

Fonte: Elaborazione su dati Agenzia del Territorio

Nell'ultimo periodo, si registra, inoltre, un consistente spostamento dell'attività di mercato dai capoluoghi verso il resto della provincia, con particolare riferimento alle aree metropolitane adiacenti le principali realtà urbane del Nord (Andreussi, Festa, 2006). Escludendo le zone turistiche, le cause di tale fenomeno vanno rintracciate, da un alto, nel notevole aumento dei prezzi delle abitazioni nei centri maggiori, che ha spinto a cercare soluzioni alternative nei comuni limitrofi, e, dall'altro, nella disponibilità di nuove costruzioni nei comuni dell'hinterland, caratterizzate, per altro, da soluzioni tipologiche più indipendenti e insistenti su un territorio a più bassa densità. Tale effervescenza di mercato è legata ad altri due fenomeni molto importanti: la tendenza marcata alla proprietà privata della prima casa e la progressiva erosione del mercato dell'affitto.

La politica a favore della proprietà della casa è quella che più ha caratterizzato l'Italia, sin dal dopoguerra. Essa è risultata coerente alle esigenze di risparmio e investimento sicuro delle famiglie e dei proprietari di immobili e terreni ed è stata favorita, più di recente, dalla forte flessione dei rendimenti nei mercati finanziari e dall'elevata appetibilità dei mutui ipotecari, il cui tasso d'interesse passivo ha raggiunto i livelli minimi riscontrati, in Italia, solo negli anni ' 70 del '900. Ad esempio, secondo I'ISTAT ${ }^{5}$, nel 2003, una famiglia su 10 pagava un mutuo e la percentuale sale al 13 per cento se si considerano le sole famiglie in abitazioni di proprietà. L'esborso, pur rappresentando un investimento, non includibile quindi nel calcolo della spesa per consumi, costituisce un'uscita piuttosto consistente del bilancio familiare. La rata media mensile pagata per il mutuo dalle famiglie in proprietà era pari a 403,80 euro (Tabella 2), il 4,8 per cento in più dell'anno precedente: l'importo più elevato si osservava nel Nord-est $(435,91$ euro al mese) quello più basso nelle Isole (318,73 euro).

Tabella 2 - Canone medio mensile di locazione pagato dalle famiglie che vivono in affitto e rata media mensile pagata per mutui dalle famiglie che vivono in abitazioni di proprietà.

\begin{tabular}{|c|c|c|c|c|c|c|}
\hline VOCI DI SPESA & Nord-ovest & Nord-est & Centro & Sud & Isole & Italia \\
\hline Affitto & 304,65 & 333,1 & 341,04 & 218,74 & 228,81 & 288,37 \\
\hline Mutuo & 419,26 & 435,91 & 419,88 & 345,19 & 318,73 & 403,8 \\
\hline
\end{tabular}

per ripartizione geografica - Anno 2003 (in euro)

Fonte: ISTAT, I consumi delle famiglie, Anno 2003, Serie Annuari

\footnotetext{
${ }^{4}$ Dal secondo dopoguerra ad oggi, l'analisi degli investimenti in costruzioni, ci mostra sei cicli edilizi. Con l'esclusione del primo ciclo, caratterizzato da una crescita straordinaria durata quattordici anni, dal 1951 al 1964, dovuta alla ricostruzione e al miracolo economico, la fase espansiva che stiamo attraversando è la più lunga mai riscontrata. Per un approfondimento sui cicli edilizi in Italia Cfr. Ferracuti, Marcelloni, 1982; Secchi, 1984.
}

\footnotetext{
${ }^{5}$ L'Istituto Nazionale di Statistica è un ente di ricerca pubblico. Presente in Italia dal 1926, è il principale produttore di serie statistiche ufficiali.
} 
Sempre dallo stesso tipo di indagine si ricava, ad esempio, che in Piemonte la percentuale della spesa media delle famiglie, che riguarda l'abitazione, era, nel 2003, pari al $24,34 \%$, contro un $14,69 \%$ che era destinato all'altra voce altrettanto importante, ossia ai trasporti. Spesa e reddito familiare non sono ovviamente la stessa, cosa, considerata la presenza dei risparmi e degli investimenti, di cui l'abitazione fa parte ${ }^{6}$.

In particolare, per ciò che concerne il mercato dell'affitto e secondo le stime CRESME ${ }^{7}$, dal 1991 al 2002, in Piemonte, tale quota è scesa dal 37 al 25\%; ancora più marcata è stata la diminuzione nei Comuni capoluogo della regione, con un record negativo per Torino, che ha portato il patrimonio in affitto dal $46 \%$ al $27 \%$ sul totale dello stock abitativo.

II forte impulso fornito dall'andamento generale del mercato immobiliare non ha fatto che aumentare la quantità di alloggi disponibili per l'acquisto, restringendo il mercato delle locazioni e rendendo più costoso, anche a causa del costante incremento dei valori dei canoni, l'accesso alla prima casa. La proprietà dell'immobile presuppone, infatti, una solvibilità duratura, capace di far fronte, sia agli oneri finanziari, che alle spese di manutenzione ordinaria e straordinaria. Le famiglie a basso reddito non sono in grado di sostenere tali costi, in particolare in una fase economica caratterizzata dalla flessione dei salari reali medi e da condizioni di lavoro più precarie. La politica della proprietà risponde infatti sempre di meno alla crescente necessità di flessibilità legata all'organizzazione del mercato del lavoro e a nuovi modelli di convivenza. Allo stesso tempo, la decisione di cambiare residenza, per accettare un nuovo lavoro, è condizionata dalla struttura dei mercati immobiliari, considerato che la spesa connessa all'abitazione principale - rate di mutuo o affitto - rappresenta tipicamente, come già sottolineato, la maggior quota del budget familiare. In particolare, gli elevati costi di trasloco e le spese legate alla compravendita fanno aumentare i costi di mobilità e generano inefficienze nel sistema economico. Con il risultato che la popolazione appare eccessivamente stanziale e legata al proprio territorio di origine e il sistema economico risulta ingessato.

L'edilizia residenziale pubblica, con risorse progressivamente decrescenti, non è stata certamente all'altezza di soddisfare i bisogni abitativi della parte di popolazione esclusa dal mercato; essa si è limitata, negli ultimi anni, a tamponare il disagio a fronte di un incremento del numero di famiglie sotto stress abitativo. Basti ricordare che, dal 1995, a livello nazionale, non sono stati più stanziati fondi destinati all'attività costruttiva a carattere sociale. L'intervento pubblico si è limitato alla sola erogazione dei sussidi alle famiglie, tramite il Fondo Nazionale per il Sostegno alla Locazione, istituito con la Legge 431 del 1998. Secondo il SICET (Sindacato Inquilini Casa e Territorio), gli appartamenti di edilizia pubblica sono in Italia solo 720 mila, quando ne occorrerebbero due milioni per colmare l'intero fabbisogno, mentre in Francia, con una popolazione pari a quella italiana, esistono ben tre milioni e mezzo di abitazioni cosiddette sociali. II patrimonio pubblico è stato, per altro, caratterizzato, nel tempo, da profili di scarsa qualità - dal punto di vista della vita utile e delle condizioni di efficienza, di manutenzione, ma anche e soprattutto in termini di localizzazione urbana e accessibilità - di modo che, il binomio basso costo-scarsa qualità si è trasformato in una sorta di luogo comune,

\footnotetext{
${ }^{6}$ Non dimentichiamo che l'opzione affitto-proprietà mette in gioco le scelte patrimoniali, oltre che occupazionali ed esistenziali del nucleo familiare stesso, anche se composto da una persona sola. Al centro della discussione va posta, dunque, la capacità di consumo e la spesa destinata dai nuclei familiari all'abitazione principale; in altre parole, la capacità, in termini di reddito reale, di raggiungere un adeguato rapporto tra prezzo dell'immobile - considerando eventualmente l'onere finanziario in termini di quota d'interesse - o affitto e reddito familiare. In altre parole, si tratta del costo dei servizi abitativi per il nucleo familiare, per chi non gode di un'abitazione già propria o paga un finanziamento per poterne fruire. Indicativamente e un po' in tutto il mondo, si assume che tale rapporto non debba superare il $30 \%$.
}

${ }^{7}$ CRESME (Centro Ricerche Economiche Sociali di Mercato per I'Edilizia e il Territorio) 
di verità, quanto mai difficile da contestare. Permangano, inoltre, come risaputo, fenomeni di marginalità sociale, a livello territoriale, legati alla struttura del parco abitativo pubblico e alla sua concentrazione in determinate aree urbane.

Com'è noto, i promotori immobiliari e l'industria delle costruzioni non sono interessati a promuovere interventi di edilizia residenziale caratterizzati da bassi costi e da prezzi contenuti, al di sotto della media di mercato e l'intervento diretto dello Stato trova spazio proprio perché volto a tutelare le categorie sociali più svantaggiate. La forte flessione degli investimenti in edilizia residenziale sovvenzionata ha determinato, di conseguenza, una situazione di predominio dell'offerta privata di abitazioni sul libero mercato, sia della proprietà che dell'affitto.

Erosione della locazione e stagnazione dell'edilizia pubblica appaiono dunque come fenomeni particolarmente gravi nell'area metropolitana torinese, dove, di fatto, l'intensa attività di trasformazione urbana e il flusso degli investimenti hanno prodotto fortissimi incrementi di prezzo, ma anche importanti mutamenti nella composizione sociale dei residenti e nelle destinazioni d'uso. Per ciò che concerne i prezzi, nella Tabella che segue, è possibile osservarne gli incrementi negli ultimi tre anni.

Tabella 3 - Prezzi medi di compravendita di abitazioni nuove e usate dal 2003 al 2005 (euro al mq.)

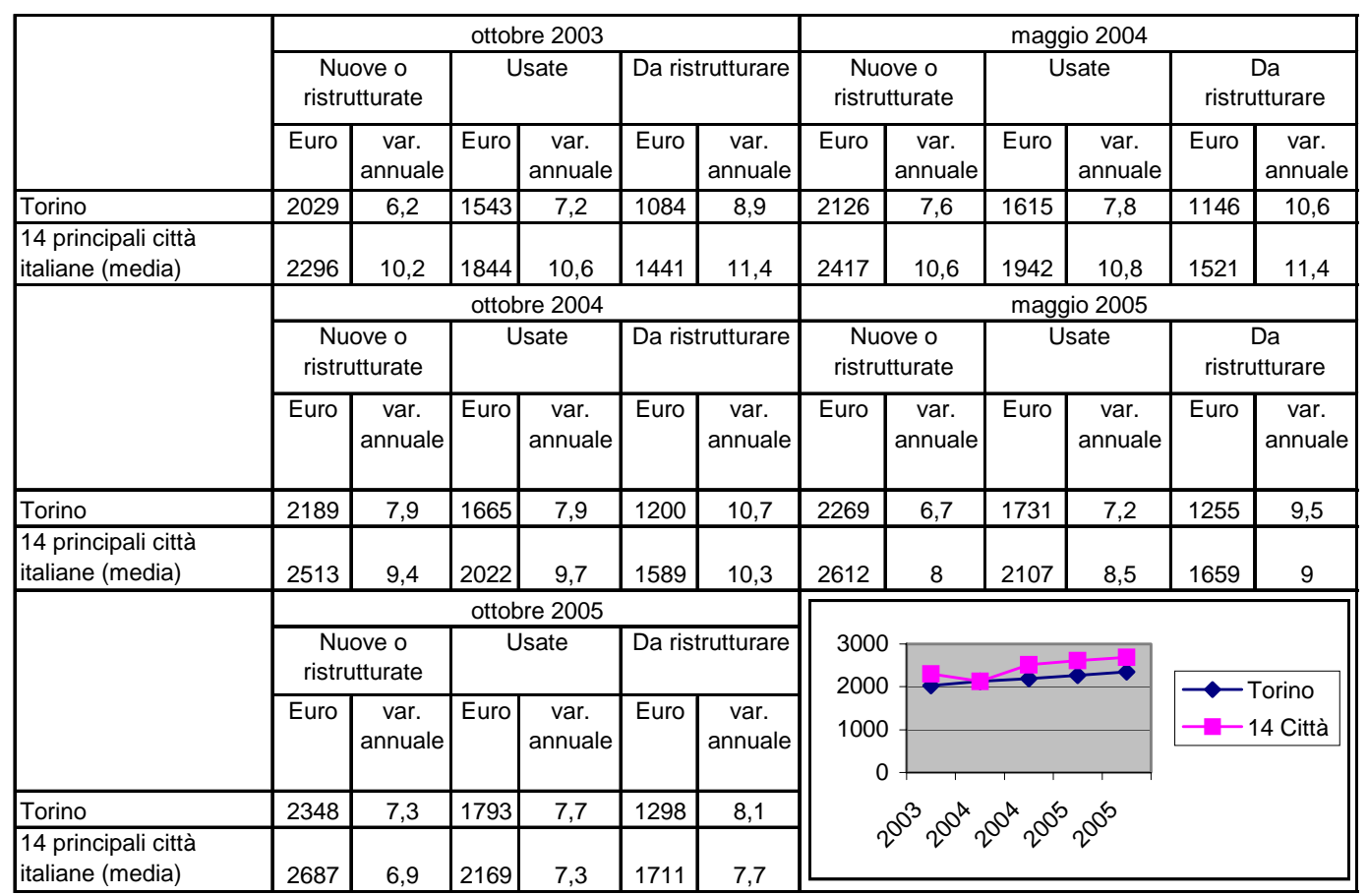

Fonte: Nomisma, Osservatorio sul Mercato Immobiliare, Quadrimestri diversi

Si nota immediatamente che Torino appare sempre al di sotto della media delle 14 principali città italiane e che, mentre il mercato immobiliare sembra subire, in generale, un lieve rallentamento degli indici di crescita, l'edilizia residenziale torinese sembra non aver evidenziato tendenze significativamente divergenti. Essa appare piuttosto in fase di assestamento. A determinare questa situazione contribuisce sicuramente la notevole quantità di mq. di abitazioni nuove immesse recentemente sul mercato. Esse rispondono infatti, prima di 
tutto, a una domanda di miglioramento dello standard abitativo attuata tramite l'investimento (Bravi i Giaccaria, 2006). II Grafico che segue mostra invece come il segmento delle nuove abitazioni evidenzi un andamento dei prezzi, in termini costanti, che, a sua volta, delinea un ciclo che si dispiegherà completamente, all'incirca, nell'arco di 15 anni; i prezzi non hanno in effetti ancora riguadagnato, in termini costanti, i valori raggiunti durante il picco del 1992.

\section{Grafico 1 - Prezzi correnti e costanti (euro al mq.) di abitazioni nuove a Torino dal 1992 al 2005}

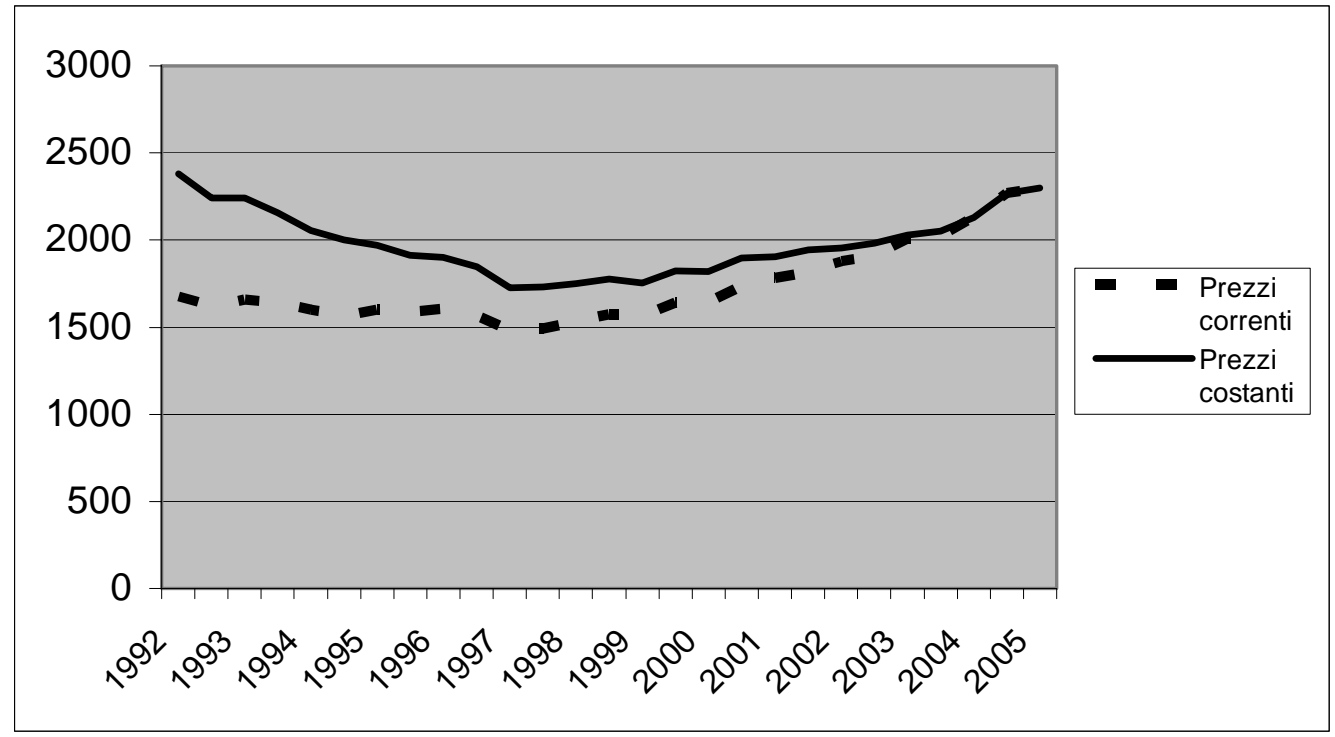

Fonte: elaborazione su dati Nomisma, Anni diversi

I movimenti di popolazione accompagnano poi i fenomeni delineati sino a questo punto. A fronte di un saldo demografico naturale negativo, si assiste infatti a un consistente flusso di popolazione immigrata di origine extra-comunitaria verso l'interno e a un cospicuo flusso di nuclei familiari giovani verso l'esterno, diretti, in particolare, verso i comuni dell'area metropolitana e, più di recente, anche verso i restanti comuni della Provincia. La Tabella che segue cerca di mettere in evidenza questo fenomeno.

Tabella 4 - Movimenti di popolazione a Torino tra il 2001 e il 2004

\begin{tabular}{|l|c|c|c|c|c|c|c|c|}
\hline Movimenti popolazione & \multicolumn{2}{|c|}{2001} & \multicolumn{2}{c|}{2002} & \multicolumn{2}{c|}{2003} & \multicolumn{2}{c|}{2004} \\
\hline \multicolumn{1}{|c|}{ Immigrati } & $\mathrm{N}^{\circ}$ & $\%$ & $\mathrm{~N}^{\circ}$ & $\%$ & $\mathrm{~N}^{\circ}$ & $\%$ & $\mathrm{~N}^{\circ}$ & $\%$ \\
\hline Estero & 5354 & 22,55 & 5288 & 24,38 & 15846 & 52,99 & 11578 & 39,92 \\
\hline Altra provenienza & 18392 & 77,45 & 16404 & 75,62 & 14060 & 47,01 & 17422 & 60,08 \\
\hline Totale & 23746 & 100 & 21692 & 100 & 29906 & 100 & 29000 & 100 \\
\hline \multicolumn{1}{|c|}{ Emigrati } & $\mathrm{N}^{\circ}$ & $\%$ & $\mathrm{~N}^{\circ}$ & $\%$ & $\mathrm{~N}^{\circ}$ & $\%$ & $\mathrm{~N}^{\circ}$ & $\%$ \\
\hline Area metropolitana & 9367 & 40,56 & 9010 & 39,63 & 3966 & 18,50 & 4527 & 20,10 \\
\hline Altre destinazioni & 13728 & 59,44 & 13728 & 60,37 & 17475 & 81,50 & 17992 & 79,90 \\
\hline Totale & 23095 & 100 & 22738 & 100 & 21441 & 100 & 22519 & 100 \\
\hline Saldo naturale & -1919 & \multicolumn{2}{|c|}{-3377} & \multicolumn{2}{c|}{-4049} & -2893 \\
\hline Totale abitanti & \multicolumn{2}{|c|}{865263} & \multicolumn{2}{c|}{896918} & \multicolumn{2}{c|}{902910} & \multicolumn{2}{c|}{902342} \\
\hline
\end{tabular}

Fonte: Elaborazione su dati Comune di Torino, Notiziario di statistica, Anni diversi 
Una nuova pressione sul mercato abitativo viene infatti esercitata dalla popolazione immigrata extra-comunitaria che alimenta sicuramente comportamenti speculativi da parte dei proprietari di immobili e che non fa che aumentare la quota di domanda esclusa dal mercato. Un numero sempre più ampio e diversificato di categorie sociali è quindi esposto, non soltanto a Torino, ma anche nei piccoli comuni della Provincia, a fenomeni di disagio abitativo, non essendo in grado di accedere al mercato privato.

\section{Le aree di trasformazione urbana: il peso dell'edilizia residenziale}

Grazie all'interramento della rete ferroviaria cittadina e alla riqualificazione delle aree industriali abbandonate, Torino è rientrata in possesso di oltre due milioni di metri quadrati del suo territorio originario. II progetto principale era, come risaputo, caratterizzato dalla cosiddetta area della "Spina Centrale", posta lungo la linea ferroviaria e in direzione Nord-Sud, che interessava quattro comparti (Spina 1, 2, 3 e 4$)^{8}$ e il grande asse viario di collegamento, realizzato al di sopra del passante ferroviario, che dalla periferia Est giunge sino al centro della Città. L'esistenza di grandi stabilimenti industriali abbandonati e di impianti ormai inattivi o sotto-utilizzati ha rappresentato, per dimensioni e localizzazione, una straordinaria occasione di rinnovo urbano, in grado di mutare la stessa geografia della Città, creando nuove centralità ed elementi di ricomposizione urbana in zone che hanno costituito, nel passato, una frattura nel tessuto consolidato (Dansero et al., 2003).

In termini di superficie totale sono stati proprio i PRIU (Piani di Riqualificazione Urbana) ad avere il maggiore rilievo quantitativo tra gli strumenti attuativi, seguiti dalle trasformazioni convenzionate, come le ATS (Aree di Trasformazione a Servizi) e le ZUT (Zone Urbane di Trasformazione). La maggior parte degli strumenti ha interessato prevalentemente il settore residenziale, tranne nel caso dei Piani Particolareggiati (PP), orientati per lo più al settore produttivo. Dal punto di vista della densità territoriale, gli indici più elevati sono quelli relativi a Programmi Integrati, PP e ZUT; i più bassi indici di densità interessano invece le ATS.

\footnotetext{
${ }^{8}$ L'intera "Spina Centrale" è stata suddivisa in quattro principali ambiti di intervento. Viene abitualmente identificato come "Spina 1" l'ambito Sud, tra i corsi Lione, Mediterraneo, Rosselli e Tirreno, ove, un tempo, erano localizzate le Officine Materiale Ferroviario (Materferro) dell'industria automobilistica Fiat: nel complesso, si tratta di un'area pari a 142.000 mq. Il comparto denominato "Spina 2" è, invece, quello delle aree industriali abbandonate, un tempo occupate dalle Officine Grandi Riparazioni, dalla Nebiolo e dalla Westinghouse; è delimitato dai corsi Castelfidardo e Ferrucci, da un lato e da via P.C.Boggio, dall'altro: nel complesso, si tratta di un'area di $340.000 \mathrm{mq}$. Essa verrà servita dalla nuova stazione ferroviaria di "Porta Susa", di interscambio tra passante e metropolitana, ancora in fase di ristrutturazione. In quest'area sono già stati avviati diversi altri grandi interventi: come il cosiddetto raddoppio del Politecnico di Torino, la nuova Biblioteca Civica centrale e sala teatrale, polo espositivo, ecc. Quello della cosiddetta "Spina 3" è l'ambito di maggiore trasformazione del PRG, pari a oltre un milione di mq. L'area è suddivisa in sette comprensori e in quindici sub-comprensori: Michelin, Valdocco, ove trova collocazione l'Environment Park, Vitali, Valdellatorre, Paracchi, Savigliano e Ingest. II Programma di riqualificazione urbana di "Spina 4" interessa, invece, alcune aree industriali alla periferia Nord della Città. Va poi ricordato che, lungo la direttrice Sud, troviamo il centro polifunzionale del "Lingotto". L'ex stabilimento della Fiat è stato da tempo trasformato e, al suo interno, trovano oggi spazio un centro congressi, l'Auditorium, un Hotel della catena "Le Méridien", la galleria commerciale "8 Gallery", una multisala cinematografica, un supermercato e la Pinacoteca "Giovanni e Marella Agnelli" (Cfr. Airaldi et al., 2004; Talarico, 2003-04).
} 
Immagine 1 - Alcune vedute delle "Spine"
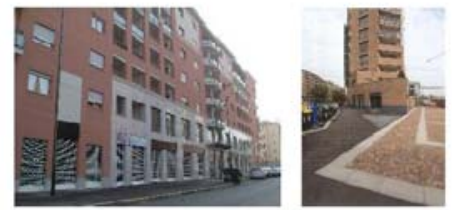

Spina
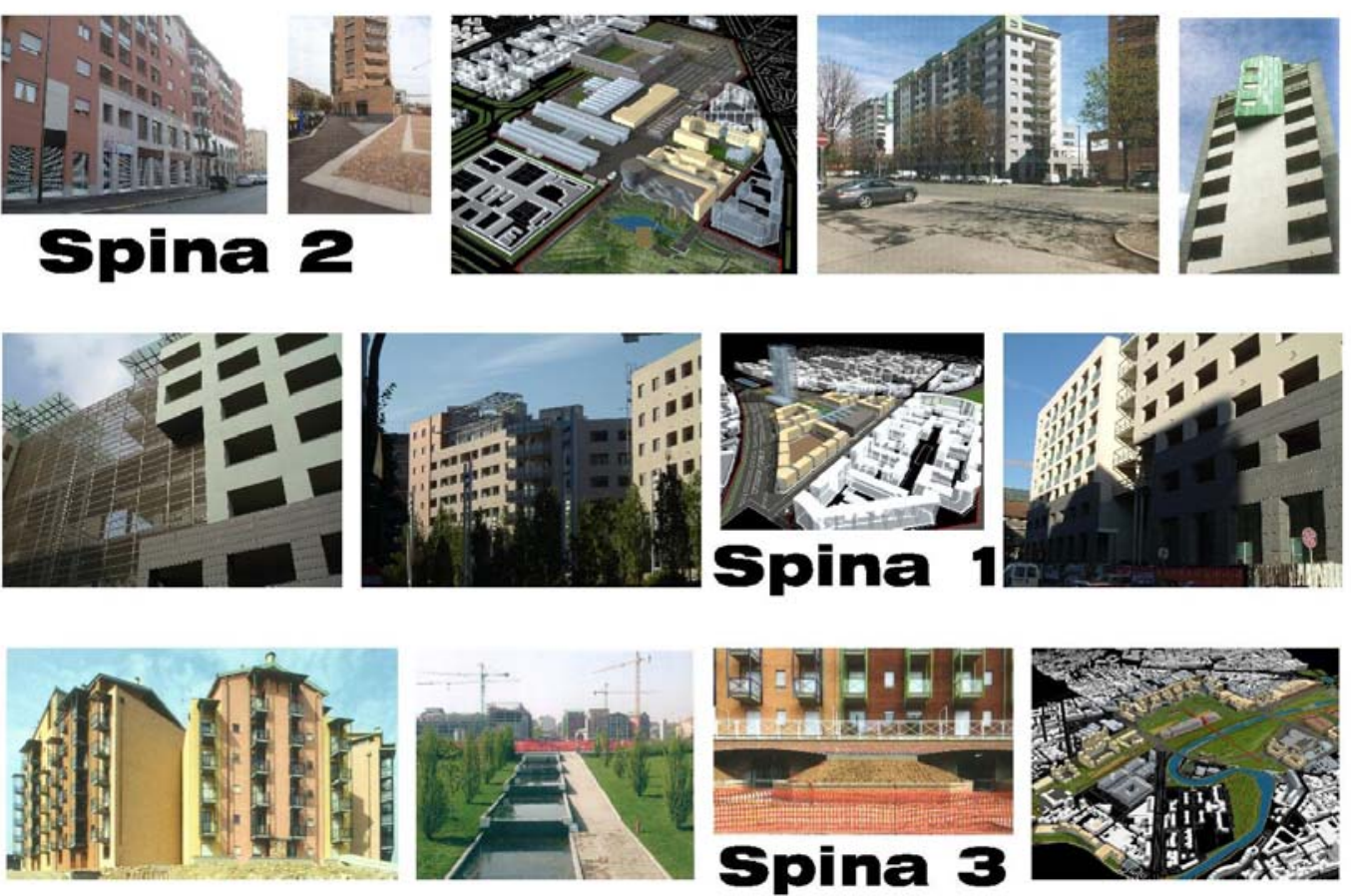

Tabella 5 - Destinazioni d'uso in mq. di SLP nelle aree di trasformazione delle "Spine"

\begin{tabular}{|l|c|c|c|c|c|c|c|}
\hline & \multicolumn{7}{|c|}{ Destinazioni d'uso previste } \\
\cline { 2 - 8 } & sup. t. & \multicolumn{2}{|c|}{ s.l.p. residenziale } & \multicolumn{2}{c|}{$\begin{array}{c}\text { s.l.p. } \\
\text { altre attività }\end{array}$} & $\begin{array}{c}\text { s.l.p. } \\
\text { totale }\end{array}$ & nuovi abitanti \\
\hline Spina 1 & 163336 & 51321 & 67,67 & 24514 & 32,33 & 75835 & 1509 \\
\hline Spina 2 & 147228 & 39392 & 42,98 & 52267 & 57,02 & 91659 & 848 \\
\hline Spina 3 & 1002956 & 348375 & 59,60 & 236167 & 40,40 & 584542 & 10246 \\
\hline Spina 4 & 149953 & 77492 & 88,80 & 9778 & 11,20 & 87270 & 2279 \\
\hline
\end{tabular}

Fonte: Comune di Torino

Si tenga presente che, nel complesso, nell'arco degli ultimi 15 anni, sono stati riqualificati e riutilizzati più di tre milioni di metri quadri di aree industriali dismesse. Dopo una prima fase di disinteresse dovuto alla mancanza di condizioni sociali, economiche e politiche favorevoli, si è passati, infatti, a considerare questi siti come potenziali risorse; il problema dell'individuazione delle destinazioni d'uso è stato al centro della riflessione del Piano, ma anche della contrattazione tra operatore pubblico e privato. E' stato osservato (L'Eau Vive, Comitato Giorgio Rota, 2004) come l'Amministrazione Comunale sia stata, in questi ultimi anni, sottoposta frequentemente alle pressioni dei costruttori affinché venissero ulteriormente ampliate le quote di superfici previste per abitazioni, rispetto a quelle previste per il terziario; ciò si è verificato anche in corrispondenza di aree, come appunto quelle delle grandi trasformazioni, vicino alle stazioni, che dovrebbero caratterizzarsi proprio per la loro vocazione terziaria, ospitando servizi di rango metropolitano e regionale. Analizzando nello specifico la 
situazione delle quattro "Spine" si nota infatti come la superficie residenziale oscilli tra il 40 e il $90 \%$ di quella totale edificabile (Immagine 2 ).

Immagine 2 - Localizzazione della "Spina centrale" e dei quattro principali ambiti

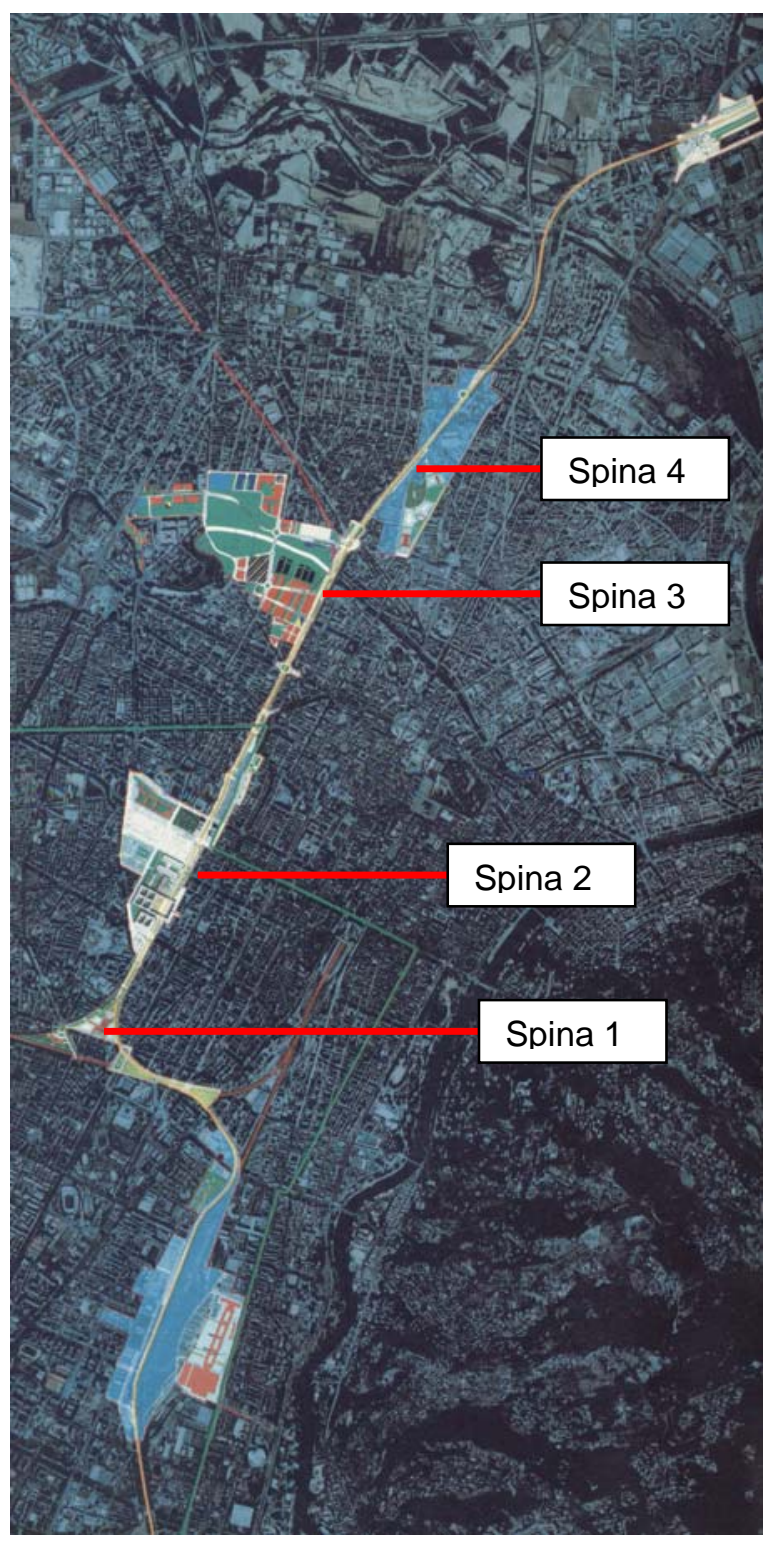

Fonte: Comune di Torino, Divisione Edilizia e Urbanistica, Progetti di riassetto urbano 
E' utile, al fine che ci è proposti, ripercorrere brevemente la vicenda del comparto più ampio, quello di "Spina 3". La sua approvazione è avvenuta con l'Accordo di Programma adottato dal Presidente della Giunta Regionale nel maggio 1999 in variante al Piano Regolatore, che assegnava 49 miliardi di contributi pubblici per le bonifiche, le demolizioni e altre opere di urbanizzazione riguardanti le aree a destinazione pubblica che gli operatori privati cedevano al Comune. L'attuazione della riconversione è iniziata con la realizzazione del parco tecnologico e ambientale "Environment Park", all'interno del comprensorio "Valdocco". A tale proposito era stata creata una società di gestione a carattere prevalentemente pubblico, legata alle stesse logiche della trasformazione, che implicava la bonifica e il recupero ambientale delle aree inquinate dalle industrie pesanti (Curwell i Lombardi, 1999). La prima fase di tale intervento si conclude nel 2001 e nel 2002 viene approvata la variante n.35 del PRG che si occupa di definire meglio i quattro ambiti delle "Spine", mentre nel 2003 il Consiglio Comunale stabilisce, sulla base dei programmi olimpici, di localizzare il "Villaggio Media" nei comprensori "Vitali" e "Michelin Nord" di "Spina 3". Lo stesso anno si decide anche di acquistare 280 alloggi da destinare alla locazione permanente a canone convenzionato. Viene pure presentato il progetto della chiesa del Santo Volto e del complesso della curia metropolitana, a firma dell'architetto Mario Botta e che "è forse l'emergenza architettonica più rilevante" (Spinelli, 2005-06, p.51) dell'ambito "Spina 3". Nel 2004 viene aggiudicata la progettazione del cosiddetto "Parco Dora" e, l'anno successivo, viene approvato il progetto preliminare. Viene altresì stabilita la destinazione residenziale post-olimpica dei fabbricati già realizzati, riservandone una certa quota a categorie sociali svantaggiate. Occorre sottolineare che la variante del 2001 modificava le consistenze definite nel 1998 dal primo Accordo di Programma al fine di consolidare nell'area la presenza di attività economiche; un'ulteriore variazione si registrerà con la variante del 2003 (Tabella 6). E' da notare la modificazione, nel corso del tempo, delle superfici terziarie e produttive, con la sostanziale tenuta di quelle a destinazione residenziale e commerciale e l'aumento di quelle destinate a servizi.

Tabella 6 - Destinazioni d'uso nelle tre fasi del PRIU

\begin{tabular}{|l|c|c|c|}
\hline \multirow{2}{*}{ Destinazioni d'uso } & \multicolumn{3}{|c|}{ Varianti } \\
\cline { 2 - 4 } & 1998 & 2001 & 2003 \\
\hline Residenza & 352971 & 333623 & 348375 \\
\hline $\begin{array}{l}\text { ASPI (Attività di servizio } \\
\text { per Persone e Imprese) }\end{array}$ & 27272 & 40280 & 53500 \\
\hline Attività terziarie & 13580 & 7700 & 3320 \\
\hline $\begin{array}{l}\text { Attività commerciale } \\
\text { grande distribuzione) }\end{array}$ & 18000 & 18000 & 18000 \\
\hline $\begin{array}{l}\text { Euro Torino 1 (congressi, } \\
\text { università) }\end{array}$ & 80998 & 41700 & 73052 \\
\hline $\begin{array}{l}\text { Euro Torino 2 (ricerca, } \\
\text { produzione, terziario) }\end{array}$ & 93233 & 143645 & 89295 \\
\hline Totale diritti edificatori & 586054 & 584948 & 585542 \\
\hline
\end{tabular}

Fonte: Spinelli, 2055-06, su dati Comune di Torino

Ciò che vale la pena considerare riguarda tuttavia il risultato di tale trasformazione: è previsto che, nel 2011, "Spina 3" ospiterà circa diecimila nuovi abitanti distribuiti su più di quattromila nuovi appartamenti, mentre le attività commerciali e terziarie occuperanno circa settemila persone. L'assetto della proprietà dei suoli risulterà completamente modificato: al momento della chiusura degli stabilimenti, si registravano pochi proprietari (CimiMontubi, 
Michelin, Fiat, Savigliano e Paracchi) di enormi parcelle fondiarie a edificato estensivo e totalmente in disuso (Immagine 3), mentre, al termine della trasformazione, si assisterà a una quota privata più concentrata, su un edificato molto più denso - che testimonia da sola il processo di valorizzazione fondiaria - a riscontro di una presenza di suolo pubblico comunale molto consistente, con i $400.000 \mathrm{mq}$. destinati al parco della Dora.

\section{Immagine 3 - "Spina 3" - Schema di suddivisione delle proprietà fondiarie all'inizio del processo urbano di trasformazione}

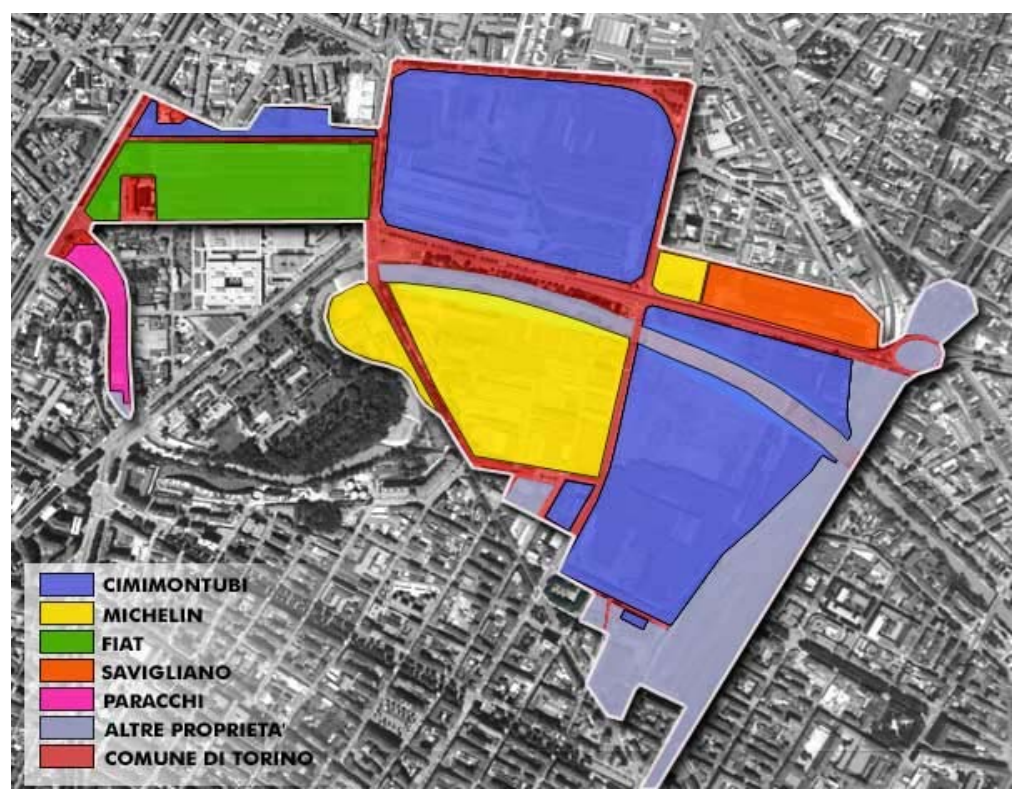

Fonte: www.cimimontubi.it

Si è trattato, in altre parole, di un'area troppo vasta, troppo radicata e, per certi aspetti troppo appetibile perché si sia potuto concepirne la metamorfosi senza il concorso dei soggetti privati (Spinelli, op. cit., p.93), senza puntare sulla valorizzazione dei suoli, attuata tuttavia con margini di rischio d'impresa assai bassi. Non dimentichiamo che il settore residenziale ha beneficiato di una congiuntura particolarmente favorevole e di una forte crescita dei prezzi nell'ultimo quinquennio. II PRIU ha funzionato proprio perché ha dato voce alle richieste degli operatori economici, che erano disposti a impiegare il capitale proprio, a fronte però di un dialogo piuttosto aperto con l'Amministrazione, spesso in deroga dai vincoli imposti dai Regolamenti edilizi sull'intero territorio comunale. La ricerca di una mixité funzionale, portatrice di caratteri di urbanità, è stata comunque, sin dall'inizio, una delle discriminanti di "Spina 3". Tale obiettivo risulta premiante per i soggetti coinvolti e dal punto di vista del mercato (Corsico, Perletti, 2000). La presenza di una multisala cinematografica e di un ipermercato, assieme ad attività di ristorazione e spazi per uffici, ne ha incrementata sicuramente l'attrattività, migliorandone anche l'immagine. L'offerta edilizia è poi risultata sufficientemente differenziata e adatta a intercettare i segmenti di domanda privata. Ciò a testimonianza di un ulteriore tentativo di diversificazione del rischio in una situazione ove, di norma, non vengono effettuate analisi della domanda abitativa di tipo ex-ante l'intervento. In Tabella 7 viene evidenziato, a titolo d'esempio, il rango di variazione dei prezzi all'interno del comprensorio "Valdocco" a maggio 
del 2002. Si noti che il valor medio supera, seppur di poco, quello cittadino, a testimonianza della forte appetibilità della zona.

Tabella 7 - Prezzi di vendita di abitazioni nuove all'interno del comprensorio "Valdocco" - Ambito "Spina 3" - secondo i diversi segmenti di mercato (maggio 2002 - euro/mq.)

\begin{tabular}{|l|c|}
\hline \multicolumn{2}{|c|}{$\begin{array}{c}\text { Comprensorio "Valdocco" - Ambito "Spina 3" - } \\
\text { Segmenti di mercato }\end{array}$} \\
\hline \multicolumn{1}{|c|}{ Sub-comprensorio UMI II } & Euro/mq. \\
\hline Giovani Coppie & 1859,87 \\
\hline Family & 1735,86 \\
\hline Executive & 1800,91 \\
\hline Privilege & 1776,49 \\
\hline Prestige & 2329,47 \\
\hline \multicolumn{1}{|c|}{ Sub-comprensorio UMI V } & \\
\hline Giovani Coppie & 1888,43 \\
\hline Family & 1798,99 \\
\hline Executive & 1845,56 \\
\hline Privilege & 1812,74 \\
\hline Prestige & 2368,35 \\
\hline Media Sub-comprensori & 1921,67 \\
\hline $\begin{array}{l}\text { Media Città di Torino } \\
\text { (abitazioni nuove) }\end{array}$ & 1907,26 \\
\hline Fonte: rilevazione diretta &
\end{tabular}

Fonte: rilevazione diretta ${ }^{9}$

Occorre comunque ricordare che la presenza, accanto al libero mercato, di interventi residenziali destinati all'edilizia convenzionata, è un segnale di volontà di controllo delle dinamiche di mercato; appare tuttavia come una compensazione solo parziale della quota di diritti edificatori pubblici andati persi nel corso del processo di attuazione del PRIU.

\section{Conclusioni}

II PRG fondava dunque le sue basi sul principio di trasformabilità fisica delle aree industriali abbandonate e, pur restando ancora oggi un'idea portante, consolidatasi nella pratica urbanistica, sono stati individuati altri obbiettivi, tali da modificare la città in maniera più radicale rispetto a quanto previsto dal Piano: il ripensamento del ruolo delle stazioni ferroviarie - "Porta Nuova" e lo scalo del "Lingotto" - in relazione a un nuovo progetto strategico della mobilità. In particolare, la trasformazione di "Porta Nuova", nell'ipotesi di un ridimensionamento del numero dei binari assieme all'abbassamento del piano del ferro, rappresenta la possibilità di connettere due parti di Città storicamente separate, coerentemente al quadro esistente, al fine di eliminare una delle ultime cesure presenti nel tessuto urbano torinese (Lami et al., 2006, p.108). Non solo. II secondo Piano Strategico ${ }^{10}$ pone al centro delle questioni da rilanciare:

il bisogno di superare una dimensione frammentata e urbanocentrica del sistema, guardando all'area metropolitana come la nuova dimensione della città,

\footnotetext{
${ }^{9}$ A.Talarico, Laboratorio di sintesi finale, Facoltà di Architettura, Politecnico di Torino, Anno 2001-2002

10 "Secondo piano strategico dell'area metropolitana", disponibile su: www.torino-internazionale.org
} 
poiché è solo a questa scala che gli interventi di trasformazione assumono piena valenza funzionale e qualitativa;

- i grandi progetti relativi alle infrastrutture, ai trasporti e alla mobilità, che debbono coniugare obbiettivi di riorganizzazione funzionale, competitività e qualificazione ambientale;

- il tema dello sviluppo economico, interpretato in un'ottica dinamica ed evolutiva; il cambiamento non va inteso come una rinuncia alla vocazione industriale locale, ma come produzione con forti contenuti immateriali basati sulla ricerca, l'innovazione e le risorse culturali, compreso il turismo;

- il tema della qualità del costruito e del paesaggio urbano, che non va ridotto a un problema di estetica della città o di parti fin troppo puntuali di essa;

- il tema della condizione abitativa, che occupa una posizione centrale tra i filoni strategici che raccordano sviluppo economico e qualità sociale; in particolare l'inserimento positivo degli immigrati nel tessuto urbano non costituisce solo un elemento fondante per una integrazione non subalterna, ma è anche condizione essenziale per promuovere una società coesa e fiduciosa, che includa le differenze culturali e che valorizzi le relazioni di solidarietà.

Quest'ultimo punto appare, come ribadito nell'Introduzione, come un obiettivo di politica sociale irrinunciabile per una Città che si sta ancora trasformando, nelle sue destinazioni d'uso, ma, soprattutto, nella sua dimensione sociale ed economica.

\section{Bibliografia}

Airaldi S., Bravi M., Roscelli R. 2004. "Trasformazione di grandi strutture: il caso dello stabilimento Fiat-Lingotto a Torino", in (a cura di) S.Stanghellini, La selezione dei progetti e il controllo dei costi nella riqualificazione urbana, Alinea, Firenze, 101-118

Andreussi C., Festa M. 2006. "Continua la crescita del mercato immobiliare nelle metropoli italiane", Consulente Immobiliare, 771, 1219-1224

Ave G. 2004. Città e strategie, Urbanistica e rigenerazione economica della città. Maggioli, Rimini

Bianchetti C. (a cura di) 2006, Torino. Metabolizzare le olimpiadi. Officina, Roma

Bravi M., Giaccaria S. 2006. "La Conjoint Analysis nelle valutazioni immobiliari". Aestimum, 48, 39-59

Castagneti S. 2002-2003. Processi di trasformazione urbana e mercato immobiliare: valutazione di fattibilità. Il caso di Spina 3. Tesi di Laurea, Relatore: Prof. F.Zorzi, I ${ }^{\mathrm{a}}$ Facoltà di Architettura, Politecnico di Torino

Corsico F., Perletti M. 2000. "Torino Spina3: un caso di continuità". Urbanistica Informazioni, $169,44-45$

Curwell S., Lombardi P. 1999. "Riqualificazione urbana sostenibile: un'analisi comparativa Torino-Salford". Urbanistica, 112, 96-103

Dansero E., Governa F., Emanuel C. (a cura di) 2003. I patrimoni industriali. Una geografia per lo sviluppo locale. Franco Angeli, Milano

Ferracuti G., Marcelloni M. 1982. La casa. Mercato e programmazione. Einaudi, Torino

Lami I., Lombardi P., Roscelli R. 2005. "Scenari di trasformazione urbana: il caso di Porta Nuova a Torino". Aestimum, 46, 107-124

Mazza L. 2004. Piani, progetti, strategie. Franco Angeli, Milano 
L'Eau Vive, Comitato Giorgio Rota. 2004, Le radici del nuovo futuro. Quinto rapporto annuale su Torino. Guerini, Torino

L'Eau Vive, Comitato Giorgio Rota. 2005. L'immagine del cambiamento. Sesto rapporto annuale su Torino. Guerini, Torino

Secchi B. 1984. Il racconto urbanistico. Einaudi, Torino

Spaziante A., Ciocchetti A. (a cura di) 2006. La riconversione delle aree dimesse: la valutazione, i risultati. Franco Angeli, Milano

Spinelli C. 2005-06. Torino/Spina 3. La trasformazione di una parte di città tra intervento pubblico e costruzione di un mercato locale. Tesi di Laurea. Relatori: Prof.ri C.Olmo, C.Bianchetti, M.Rosso, $I^{a}$ Facoltà di Architettura, Politecnico di Torino

Talarico A. 2003-04. Gli studi di fattibilità nella valutazione dei grandi progetti. Il caso dello stabilimento Fiat-Lingotto a Torino. Tesi di Laurea. Relatore: Prof.ssa M.Bravi, I Facoltà di Architettura, Politecnico di Torino 